\title{
FABRICATION OF SILVER NANOPARTICLES: USING VITEX NEGUNDO L. AGAINST CISPLATIN-INDUCED NEPHROTOXICITY IN ALBINO RAT
}

\author{
JANAKIRAMAN M \\ Department of Biochemistry, J. J. College of Arts and Science (Autonomous), Pudukkottai, Tamil Nadu, India. \\ Email: mugavaiprofmj@gmail.com
}

Received: 24 July 2018, Revised and Accepted: 23 August 2018

\begin{abstract}
Objective: This study was carried out to evaluate the fabrication of silver nanoparticles using Vitex negundo L. against cisplatin-induced nephrotoxicity in albino rat.

Methods: Synthesized silver nanoparticles of $V$. negundo L. were characterized by particle size, ultra violet (UV)-visible, Fourier transform infrared (FTIR), and X-ray diffraction (XRD) analysis. Albino rats were divided randomly into six groups of six animals each. Group I normal rats were treated with an oral dose of distilled water for 15 days. Group II rats were treated with single i.p. dose of cisplatin (16 mg/kg) on day 1 . Group III rats were treated only with an oral dose of $V$. negundo $L .(200 \mathrm{mg} / \mathrm{kg} / \mathrm{d})$ for 15 days. Group IV rats were treated only with an oral dose of silver nanoparticles of $V$. negundo L. (200 mg/kg/d) for 15 days. Group V rats were treated with an oral dose of $V$. negundo L. for 14 days after single i.p. dose of cisplatin on day 1. Group VI rats were treated with an oral dose of silver nanoparticles of $V$. negundo L. for 14 days after single i.p. dose of cisplatin on day 1. Collected blood samples and kidney tissue samples were used for biochemical, enzymatic antioxidant, and histopathological studies in all groups.
\end{abstract}

Results: The formation of silver nanoparticles was confirmed by particle size, UV-visible, and XRD analysis, and active principles were identified by FTIR analysis. Biochemical results of silver nanoparticles of $V$. negundo L.-treated groups showed the significant $(p<0.05)$ decrease in the level of creatinine, urea, and uric acid as compared to cisplatin-induced rats. The enzymatic antioxidants results of silver nanoparticles of $V$. negundo L.-treated groups showed the significant $(\mathrm{p}<0.05)$ increase in the level of glutathione reductase and significant decrease in malondialdehyde level as compared to cisplatin-induced rats. In addition, histopathological results of silver nanoparticles of $V$. negundo L-treated groups showed the ameliorated impact in cisplatin-induced rats.

Conclusion: The results of these studies were concluded that silver nanoparticles of $V$. negundo L. have profound protective effect against the adverse effects caused by cisplatin in rat kidney than V. negundo L. treated along with cisplatin in albino rat.

Keywords: Vitex negundo L., Nephrotoxicity, Silver nanoparticles, Cisplatin, Histopathology.

(C) 2018 The Authors. Published by Innovare Academic Sciences Pvt Ltd. This is an open access article under the CC BY license (http://creativecommons. org/licenses/by/4. 0/) DOI: http://dx.doi.org/10.22159/ajpcr.2018.v11i10.28721

\section{INTRODUCTION}

The kidney is an essential organ of our body because they perform many important functions in our body such as maintenance of water and electrolyte balance, regulation of blood $\mathrm{pH}$, and excretion of toxic metabolites and drugs in the form of urine. Therefore, the kidney can be considered as a major target organ for exogenous toxicants [1]. Cisplatin is one of the most potent antitumor drugs. High doses of cisplatin produce the impairment of kidney function, which is recognized as the main side effect and the most important dose-limiting factors [2]. Cisplatin is able to generate reactive oxygen species such as superoxide anion and hydroxyl radical and to inhibit the activity of antioxidant enzymes in renal tissue [3]. Renal tubular injury and death are the main pathological events in cisplatin nephrotoxicity. All of these actions eventually induce apoptosis, inflammation, necrosis, and cell death [4]. Nephrotoxicity was more found in patients who were treated with anticancer drug like cisplatin. Due to this nephrotoxicity caused by cisplatin in chemotherapy patients, the kidney cannot excrete their waste products in urine. Nephrotoxic effects may develop in glomerular and tubular epithelial cells as a result of mechanisms that disrupt normal cellular functions of mitochondria or membrane integrity and induce renal injury [5]. For the optimum function of body immunity, everyone needs nanosilver circulating in their bloodstream. Silver is one of the most naturally occurring precious metals in the world. It has been used in a wide variety of applications and some special properties such as high electrical and thermal conductivity [6]. Silver metal and its dressings have no adverse effects to the human body when it is used in reasonable amounts in our body, and it has an antimicrobial activity against some pathogens such as bacteria, viruses, fungi, and yeast [7]. Vitex negundo L. (Lamiaceae) commonly known as Nirkundi or vellanocci. It is an aromatic large shrub or small tree about $3 \mathrm{~m}$ in height with quadrangular branches and almost found throughout in India. The essential oil of $V$. negundo leaves showed the significant antifungal activity against Trichloroderma viride and Helminthosporium [8]. The extraction of the leaves showed anticancer activity against Ehrlich ascites tumor cells [9], and it also has antimicrobial activity against Escherichia coli, Klebsiella aerogenes, and Pseudomonas aerogenes [10]. However, the protective efficacy of silver nanoparticles synthesized from methanolic leaf extract of $V$. negundo L. on cisplatin-induced nephrotoxicity in albino rats was not yet carried out, so the present study was carried out to the investigation of biochemical and histopathological effects of silver nanoparticles of $V$. negundo L. on cisplatin-induced nephrotoxicity in albino rats.

\section{METHODS}

\section{Drugs and chemicals}

Cisplatin vial was used to induce nephrotoxicity procured from the medical shop, Pudukkottai, India, and the silver nitrate was purchased from Sigma-Aldrich Chemicals. All other chemicals and reagents used in the study were obtained commercially and were of analytical grade.

\section{Plant material and extract preparation}

The leaves of $V$. negundo L. were collected from Manapparai, near Trichy district, Tamil Nadu, India. The botanical identity of the plant material was authenticated by the Botanical Survey of India, 
Coimbatore, Tamil Nadu, India, and a voucher specimen of the plant material was deposited in the department under the number BSI/ SRC/5/23/2014-2015/TECH/540 for further study. V. negundo L. leaves are dried under shade and then powdered with a mechanical grinder to obtain a coarse powder. The powder was then subjected to continuous hot extraction process using Soxhlet apparatus at $60^{\circ} \mathrm{C}$ with methanol $(90 \%)$ for $72 \mathrm{~h}$. After extraction, the solvent was removed by rotary evaporator at $200^{\circ} \mathrm{C}$. The extract was concentrated and stored in desiccators.

\section{Synthesis of silver nanoparticles}

$1 \mathrm{mM}$ silver nitrate solution was prepared and used for the synthesis of silver nanoparticles. $10 \mathrm{ml}$ of the plant extract was added into $90 \mathrm{ml}$ of aqueous solution of $1 \mathrm{mM}$ silver nitrate for the reduction into $\mathrm{Ag}^{+}$ions. Immediately after the addition of silver nitrate, the color change of the leaf extract from green to dark brown was noted periodically. Then, the extract was incubated at room temperature for further incubation until $28 \mathrm{~h}$. After incubation, the silver nanoparticles were synthesized from the leaf and centrifuged at 10,000 rpm for $20 \mathrm{~min}$, and the pellet was used to further analysis [11].

\section{Characterization of silver nanoparticles}

Silver nanoparticles of $V$. negundo $\mathrm{L}$. were characterized by particle size analysis described by the method of Abd El-Rahman and Al-Jameel [12], ultra violet (UV)-visible spectrophotometer analysis by the method of Sinha et al. [13], X-ray diffraction (XRD) technique by the method of Kumari et al. [14], and Fourier transform infrared (FTIR) spectrophotometer analysis was performed by the method of Bobby et al. [15].

\section{Experimental design}

Albino rats were divided randomly into six groups of six animals each. Group I: Normal rats were treated with an oral dose of distilled water for 15 days. Group II: Rats were treated with single i.p. dose of cisplatin (16 mg/kg/d) on day 1 [16]. Group III: Rats were treated with an oral dose of $V$. negundo $\mathrm{L}$. (200 mg/kg/d) alone for 15 days [17]. Group IV: Rats were treated with an oral dose of silver nanoparticles of V. negundo L. (200 mg/ kg/d) alone for 15 days. Group V: Rats were treated with an oral dose of $V$. negundo L. from $2^{\text {nd }}$ day to $15^{\text {th }}$ day for 14 days after single i.p. dose of cisplatin on day 1. Group VI: Rats were treated with an oral dose of silver nanoparticles of $V$. negundo L. (200 $\mathrm{mg} / \mathrm{kg} / \mathrm{d}$ ) from $2^{\text {nd }}$ day to $15^{\text {th }}$ day for 14 days after single i.p. dose of cisplatin on the day 1 . Collected blood sample and kidney tissue sample were used for the studies of biochemical, enzymatic antioxidant, and histopathological studies in all groups. This study was approved by the IAEC (1416/P0/ a/11/ CPCSEA).

\section{Biochemical analysis}

The collected serum was used for the assay of urea by the method described by Marsh et al. [18], uric acid was estimated by uricase method of Ito [19], and alkaline picrate method by Brod and Sirota [20] was used to measure serum creatinine.

\section{Renal tissue marker analysis}

The prepared kidney homogenate was used for the analysis of malondialdehyde (MDA) as a measure of lipid peroxidation by the thiobarbituric acid assay method of Beuge and Aust [21], and the renal enzymatic antioxidant of glutathione reductase (GR) was done according to the procedure of David and Richard [22].

\section{Histopathological analysis}

The histopathological studies were also carried out to find the pathological changes in the different experimental groups by the method of Ochei and Kolhatkar [23].

\section{Statistical analysis}

The results were expressed as the mean value \pm standard deviation (SD). Group comparisons were performed using one-way analysis of variance test. The significant difference between normal control and experimental groups was assessed by the Student's t-test. A probability level of $<5 \%(p<0.05)$ was considered as statistically significant [24].

\section{RESULTS}

Particle size analysis

The determined average size of the particles, size distribution, and polydispersity index (PDI) of the synthesized silver nanoparticles is shown in Fig. 1. It shows that the average particle diameter is $190 \mathrm{~nm}$ and PDI is 0.29 . The average particle size and PDI revealed that the produced silver nanoparticles were monodispersed.

\section{UV-visible spectrophotometer analysis}

The nanoparticles synthesis reaction was started after the leaf extract of $V$. negundo L. was introduced into aqueous silver nitrate solution. After overnight incubation in dark room condition, the colorless reaction mixture was turned into a dark brown color due to excitation of surface plasmon resonance indicating the biotransformation of ionic silver to reduced silver. It is observed that the maximum absorbance occurs at $197 \mathrm{~nm}$ (Fig. 2). The reduction of silver ions occurred due to the watersoluble phytochemicals present in the leaf extract.

\section{FTIR analysis}

FTIR measurements were performed to identify the biomolecules responsible for capping, reducing, and stabilizing the silver nanoparticles present in the leaf extract of $V$. negundo L. The results of FTIR spectrum of silver nanoparticles of $V$. negundo $\mathrm{L}$. and its peak values with functional groups are represented in Fig. 3 and Table 1. When the silver nanoparticles of $V$. negundo L. leaf extract were passed into the FTIR, the functional groups of the active components were separated based on its peaks ratio. The results of FTIR analysis confirmed the presence of phenol, primary amines, alkyl, alkynes, carboxylic acid, aromatic compound, nitro compound, benzene, ketones, aldehyde, and bromo and fluoroalkanes compounds which showed major peaks at 3734.12, 3417.13, 2923.72,

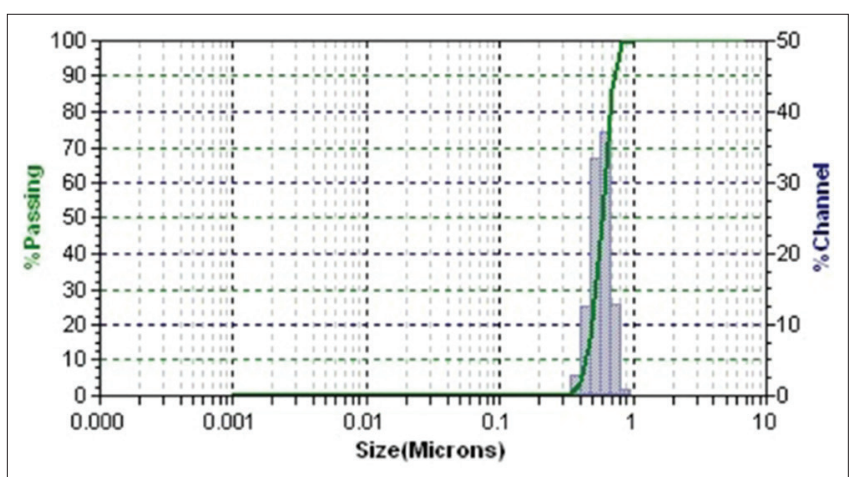

Fig. 1: Particle size distribution of silver nanoparticles of Vitex negundo $\mathrm{L}$

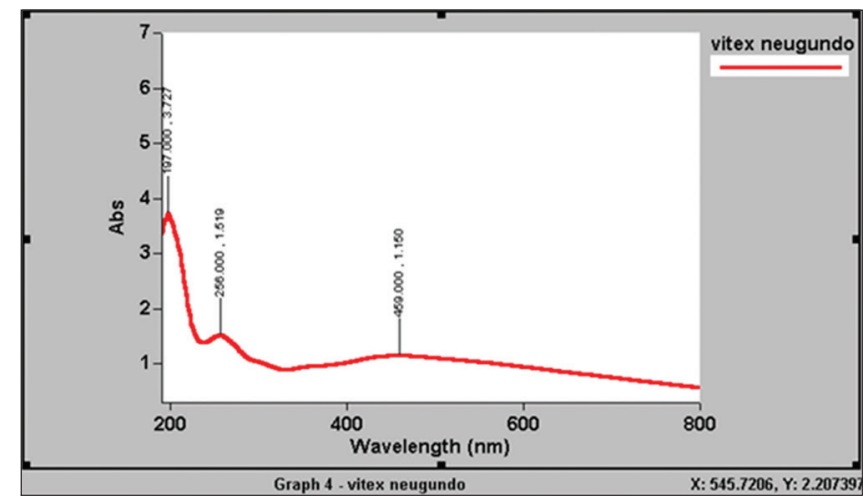

Fig. 2: Ultra violet-visible spectrum of silver nanoparticle of leaf extract of Vitex negundo $\mathrm{L}$. 
$2858.06,2320.16,1728.89,1643.48,1443.14,1378.56,1322.58$, $1258.61,1038.41,774.37$, and 613.06, respectively. The obtained results suggest that the presence of various functional groups in the synthesized silver nanoparticles of $V$. negundo $\mathrm{L}$ is shown in Fig. 3.

\section{XRD analysis}

XRD was carried out to confirm the crystalline nature of the silver nanoparticles. The XRD pattern showed numbers of Bragg reflections that may be indexed on the basis of the face-centered cubic structure of silver. A comparison of our XRD spectrum (Fig. 4) with the standard confirmed that the silver particles formed in our experiments were in the form of nanocrystals, as evidenced by the peaks at $2 \theta$ values of $14.84^{\circ}$, $21.41^{\circ}, 24.25^{\circ}, 32.12^{\circ}$ and $38.06^{\circ}$ corresponding to (111), (211), (220), (321), and (331) Bragg reflections respectively, which may be indexed

Table 1: Functional group analysis of silver nanoparticles of V. negundo L. using FTIR spectroscopy

\begin{tabular}{llll}
\hline S. No. & Peak values & Bond & Functional groups \\
\hline 1 & 3734.12 & $\mathrm{O}-\mathrm{H}$ & Phenols \\
2 & 3417.13 & $\mathrm{~N}-\mathrm{H}$ & Primary amines \\
3 & 2923.72 & $\mathrm{C}-\mathrm{H}$ & Alkyl \\
4 & 2858.06 & $\mathrm{C}-\mathrm{H}$ & Methylene \\
5 & 2320.16 & $\mathrm{C}-\mathrm{H}$ & Alkynes \\
6 & 1728.89 & $\mathrm{C}=\mathrm{O}$ & Aldehyde \\
7 & 1643.48 & $\mathrm{C}=\mathrm{O}$ & Aromatic ketones \\
8 & 1443.14 & $\mathrm{P}-\mathrm{C}$ & Aromatic compound \\
9 & 1378.56 & $\mathrm{~N}-\mathrm{O}$ & Nitro compound \\
10 & 1322.58 & $\mathrm{C}-\mathrm{O}$ & Carboxylic acid \\
11 & 1258.61 & $\mathrm{C}-\mathrm{H}$ & Meta benzene \\
12 & 1038.41 & $\mathrm{C}-\mathrm{X}$ & Fluoroalkanes \\
13 & 774.37 & $\mathrm{C}-\mathrm{H}$ & Benzene \\
14 & 613.06 & $\mathrm{C}-\mathrm{X}$ & Bromo alkanes \\
\hline
\end{tabular}

FTIR: Fourier transform infrared, V. negundo: Vitex negundo

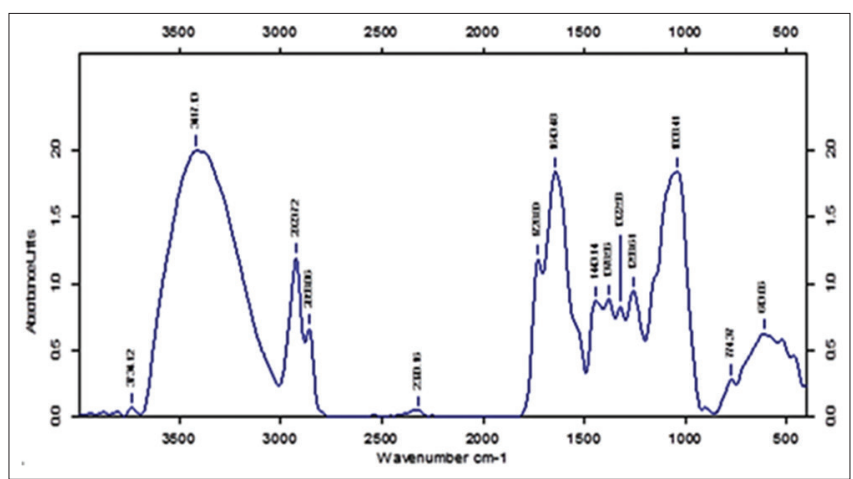

Fig. 3: Fourier transform infrared spectrum of silver nanoparticles of Vitex negundo $\mathrm{L}$.

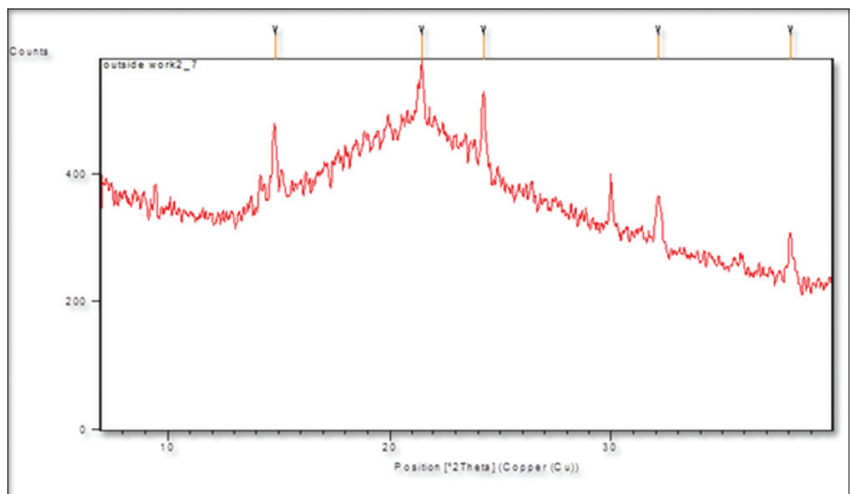

Fig. 4: X-ray diffraction spectrum of silver nanoparticles of Vitex negundo $\mathrm{L}$. based on the face-centered cubic structure of silver. XRD results clearly showed that the silver nanoparticles formed by the reduction of $\mathrm{Ag}^{+}$ions by the $V$. negundo L. leaf extract are crystalline in nature. The average particle size of silver nanoparticles synthesized by the present green method can be calculated using Debye-Scherrer equation $D=K \lambda / \beta \cos \theta$, where $\mathrm{D}$ is the crystallite size of AgNPs, $\lambda$ is the wavelength of the X-ray source $(0.1541 \mathrm{~nm})$ used in XRD, $\beta$ is the full width at half maximum of the diffraction peak, $\mathrm{K}$ is the Scherrer constant with a value from 0.9 to 1 , and $\theta$ is the Bragg angle. The average crystallite size according to DebyeScherrer equation calculated was found to be $8 \mathrm{~nm}$

Effect of silver nanoparticles of $V$. negundo L. on Biochemical parameters

Fig. 5 showed that there was a significant $(p<0.05)$ increase in the level of creatinine, urea, and uric acid of rats treated with cisplatin (Group II) as compared to normal control (Group I). In rats treated with cisplatin along with $V$. negundo L. (Group V), there was a significant $(\mathrm{p}<0.05)$ decrease in creatinine, urea, and uric acid as compared to only cisplatintreated rats (Group II). However, in rats treated with cisplatin with silver nanoparticles of $V$. negundo L. (Group VI), there were profound and significant $(\mathrm{p}<0.05)$ changes in all these serum kidney markers as compared to only cisplatin-treated rats (Group II) as well as compared to rats treated with cisplatin along with V. negundo L. (Group V), and the values were restored near to normal level which indicated the significant effect of silver nanoparticles of $V$. negundo L.

Effect of silver nanoparticles of $V$. negundo L. on renal tissue markers

The effects of silver nanoparticles of $V$. negundo L. on lipid peroxidation and renal tissue enzyme marker such as GR of the different experimental groups are depicted in Fig. 6. We found that there was a significant $(\mathrm{p}<0.05)$ increase in the level of lipid peroxidation and significant $(\mathrm{p}<0.05)$ decrease in the level of GR of rats treated with cisplatin (Group II) as compared to normal control (Group I). In rats treated with cisplatin along with $V$. negundo L. (Group V), there was a significant $(\mathrm{p}<0.05)$ decrease in lipid peroxidation and significant $(\mathrm{p}<0.05)$ increase in GR as compared to only cisplatin-treated rats (Group II). However, in rats treated with cisplatin along with silver nanoparticles of $V$. negundo L. (Group VI), there were profound and more significant $(\mathrm{p}<0.05)$ changes in lipid peroxidation, and renal enzymatic antioxidants were found as compared to only cisplatintreated rats (Group II) as well as compared to rats treated with cisplatin along with $V$. negundo L. (Group V) and the values were

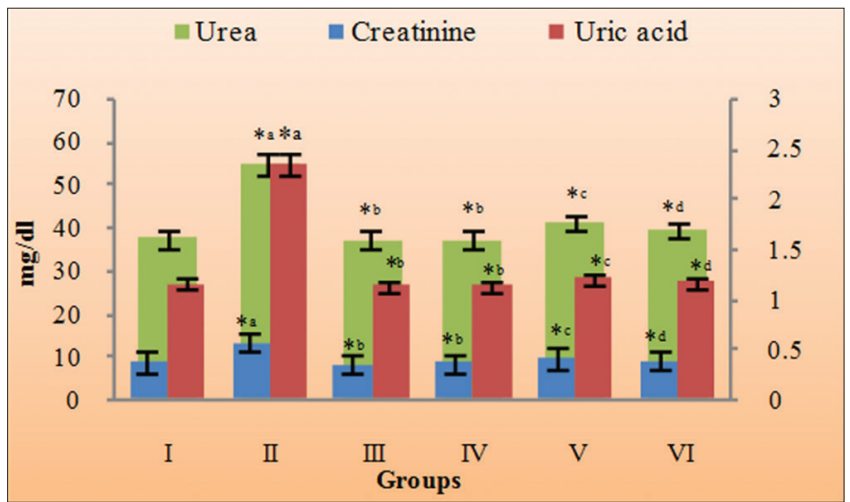

Fig. 5: Effect of silver nanoparticles of Vitex negundo L. on serum creatinine, uric acid, and urea level in the different experimental groups. All values were expressed as mean $\pm S D(n=6)$. Statistically significant of ${ }^{* a} p<0.05$ and ${ }^{* b} p<0.05$ compared to normal control Group (I), ${ }^{* c} \mathbf{p}<0.05$ compared to cisplatin-treated Group (II), and ${ }^{* \mathrm{~d}} \mathbf{p}<0.05$ compared to cisplatin-treated Group (II) and

V. negundo L. along with cisplatin-treated Group (V). Same superscript letters were not significantly $(p<0.05)$ different from each other 


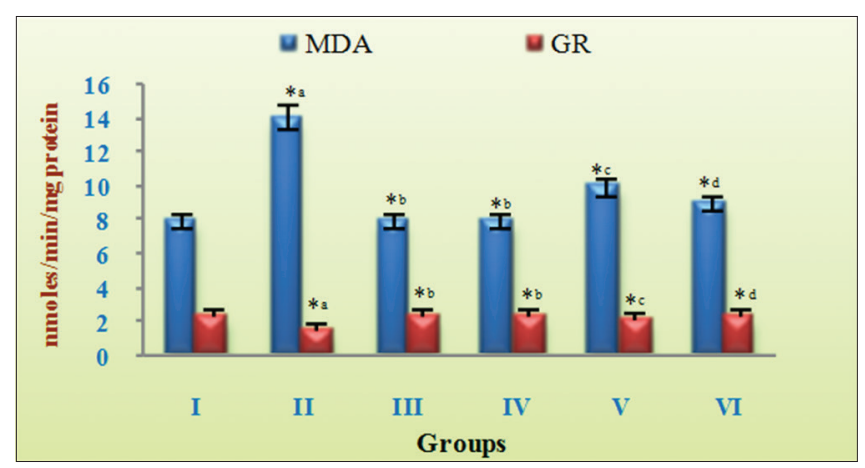

Fig. 6: Effect on silver nanoparticles of Vitex negundo L. on malondialdehyde and glutathione reductase level in different experimental groups. All values were expressed as mean $\pm S D$ $(n=6)$. Statistically significant of ${ }^{* a} p<0.05$ and ${ }^{* b} p<0.05$ compared to normal control Group (I), ${ }^{* c} \mathbf{p}<0.05$ compared to cisplatintreated Group (II), and ${ }^{* \mathrm{~d}} \mathbf{p}<0.05$ compared to Cisplatin-treated Group (II) and V. negundo L. along with cisplatin-treated Group (V), Same superscript letters were not significantly $(p<0.05)$ different from each other

restored near to normal level which indicated the profound significant effect of silver nanoparticles of $V$. negundo L. There was no significant $(\mathrm{p}<0.05)$ change in serum kidney markers, lipid peroxidation, and renal enzymatic antioxidants also observed in groups with only $V$. negundo L.-treated rats (Group III) and silver nanoparticles of $V$. negundo L. only treated rats (Group IV) as compared to normal control rats (Group I) which showed the there was no adverse effect of $V$. negundo L. and silver nanoparticles of $V$. negundo L. in albino rats.

Effect of silver nanoparticles of $V$. negundo L. on histopathological examination

The effects of silver nanoparticles of $V$. negundo L. on histopathological changes of different experimental groups are shown in Fig. 7a-f. The cortex shows normal glomeruli, tubules, interstitium, and blood vessels in the kidney of normal control rat (Fig. 7a). In cisplatintreated rat (Fig. 7b), the glomeruli vary from normal to atrophic. There is an extensive tubular necrosis characterized by sloughing of the epithelium. The tubular lumina show many desquamated epithelial cells. In V. negundo L. alone treated rat (Fig. 7c), the cortex shows normal glomeruli, tubules, interstitium, and blood vessels. There are no pathological changes in the kidney. The cortex shows normal glomeruli, tubules, interstitium, and blood vessels. There are no pathological changes also found in the kidney of rat treated with silver nanoparticles of $V$. negundo L. alone (Fig. $7 \mathrm{~d}$ ). The glomeruli are completely normal. There is a reversal of tubular necrosis. Hence, all the tubules appear normal. There is no evidence of eosinophilic hyaline casts of Tamm-Horsfall protein in the collecting ducts. The interstitium and blood vessels are normal in the kidney of rat treated with cisplatin along with $V$. negundo L. (Fig. 7e) and the kidney of rat treated with cisplatin along with silver nanoparticles of $V$. negundo L. (Fig. 7f).

\section{DISCUSSION}

The results obtained from this present work were discussed here with previous studies to support the protective efficacy of $V$. negundo L. Particle size analysis result was revealed that the monodispersed and average particle size of silver nanoparticle produced from $V$. negundo L. This results supported with previous studies of Kalainila et al., on synthesis and characterization of silver nanoparticle from Erythrina indica [25]. UV-visible result of this study proved that the silver nanoparticle producing water-soluble phytochemicals presence in the leaf extract which indicates the reducing properties of $V$. negundo L. These results were in accordance with previous studies of Shankar et al., on the rapid synthesis of $\mathrm{Au}, \mathrm{Ag}$, and bimetallic $\mathrm{Au}$ core-Ag shell nanoparticles using Neem (Azadirachta indica) leaf broth [26]. FTIR result also revealed that the presence of phenolic and flavonoid

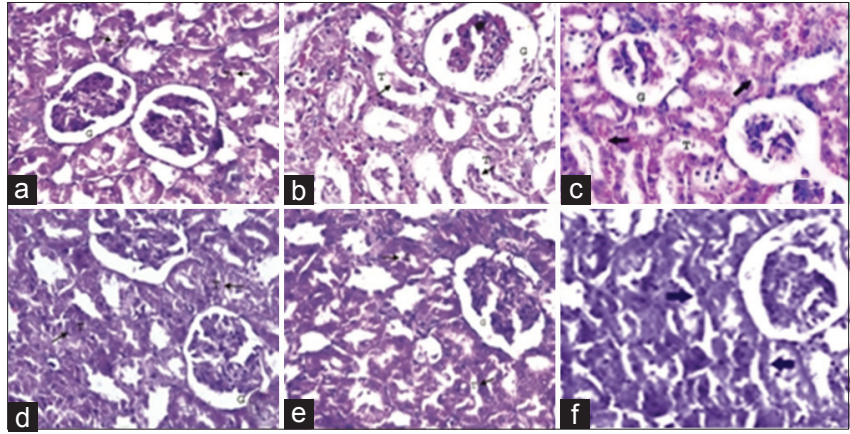

Fig. 7: (a-f) Light micrographs of renal tissue of different experimental group of rats $(\mathrm{H}$ and $\mathrm{E}, \times 400)$. (a) Normal control rats (Group I) showing the normal organization of tubular lining epithelial cells ( $T$ and arrows) and glomeruli (G). (b) Cisplatin-treated rats (Group II) showing severe congestion in glomeruli (G) and disappearance of lining epithelium in tubular cells ( $\mathrm{T}$ and arrows). (c and d) Renal sections of Vitex negundo L.-treated rats (Group III) and silver nanoparticles of $V$. negundo L.-treated rats (Group IV) showing normal organization of tubular lining epithelial cells ( $T$ and arrows) and glomeruli (G). (e) Renal sections of $V$. negundo L. treated in cisplatin-induced rats (Group V) showing regenerative changes in lining epithelium of tubules (T and arrows) and glomeruli (G). (f) Renal sections of silver nanoparticles of $V$. negundo L. treated (Group VI) in cisplatin-induced rats showing profound regenerative changes in lining epithelium of tubules (T and arrows) and glomeruli (G) as compared to Group (v) rats were treated with V. negundo L. in cisplatin-induced rats

compounds in the nanoparticles of $V$. negundo $\mathrm{L}$. which indicates the medicinal properties of their leaf extract and this result agreed with Pawar and Kamble on phytochemical screening, elemental, and functional group analysis of $V$. negundo L. leaves [27]. XRD result was also confirmed the crystalline nature of the silver nanoparticles of $V$. negundo L., and these XRD findings were supported by Amudha et al., on biosynthesis and characterization of silver nanoparticles using the aqueous extract of $V$. negundo L. [28]. Creatinine is a by-product of muscle metabolism, and it also serves as a potential marker for the assessment of renal health. Creatinine is completely removed from the blood by the mechanism of glomerular filtration as well as by proximal tubular secretion of the kidneys. If there is any defect found in the kidney, creatinine levels of blood were increased and it also increased by increased dietary intake of creatine and eating a lot of meat [29]. In mammals, the kidney serves as a vital organ for the excretion of urea from the body, and it does not only act as a carrier of waste nitrogen but also play a role in the reabsorption of water and critical ions from the excreted urine [30]. Uric acid is an end product of degradative pathway of purine metabolism which is excreted in urine. About $70 \%$ of the uric acid is daily excreted by the kidneys in human. If any defect or inflammation occurs in the kidney, uric acid level may increase which leads to gout in the body [31]. Hence, the measurement of creatinine, urea, and uric acid in blood is very important one for the assessment of renal function. This study results were revealed that there was an increased level of creatinine, urea, and uric acid in cisplatin-treated rats which is restored to normal level by silver nanoparticles of $V$. negundo L. These results were in accordance with the previous studies on protection of curcumin and curcumin nanoparticles against cisplatin-induced nephrotoxicity in male rats by Abd El-Rahman and Al-Jameel [12]. Lipid peroxidation refers to the oxidative degradation of lipids. It is the process by which free radicals "steal" electrons from the lipids in cell membranes, resulting in cell damage. The end products of lipid peroxidation are reactive aldehydes, such as MDA and 4-hydroxynonenal, and these are major bioactive marker of lipid peroxidation, due to its numerous biological activities, resembling activities of reactive oxygen species [32]. We found that there is an increased level in lipid peroxidation in cisplatin- 
treated rats, whereas lipid peroxidation level restored near to normal level in silver nanoparticles of $V$. negundo L. along with cisplatintreated rats which indicate the significant $(\mathrm{p}<0.05)$ effect of this plant extract, and these results were supported with previous studies of Abd El-Rahman and Al-Jameel on protection of curcumin and curcumin nanoparticles against cisplatin-induced nephrotoxicity in male rats [12]. GR also known as glutathione-disulfide (GSSG) reductase is an enzyme which catalyzes the reduction of GSSG to the sulfhydrylform glutathione (GSH), which is a critical molecule in resisting oxidative stress and maintaining the reducing environment of the cell. GR functions as dimeric disulfide oxidoreductase and utilizes a flavin adenine dinucleotide prosthetic group and nicotinamide adenine dinucleotide phosphate to reduce one molar equivalent of GSSG to two molar equivalents of GSH [33]. These results revealed that there is decreased level of GR in cisplatin-treated groups, whereas there is restored level of GR in rats treated with silver nanoparticles along with cisplatin which indicate the profound significant $(\mathrm{p}<0.05)$ effect of silver nanoparticles of $V$. negundo L. This results were proved with Mohamed et al., who have revealed that antioxidant and antiinflammatory activities of silver nanoparticles biosynthesized from aqueous leaves extracts of four Terminalia species [34]. Furthermore, these findings were confirmed by the renal histological results, which revealed more extensive and marked tubular and glomerular damage in the cisplatin-treated group. These findings were supported by Wahdan et al., who have reported that piceatannol ameliorates cisplatin-induced histological and biochemical alterations in rats' kidney [35]. However, in the silver nanoparticles of $V$. negundo L. and $V$. negundo L.-treated groups, renal microscopic changes were ameliorated and the renal histological architecture was almost normalized. The ameliorative effect of silver nanoparticles of $V$. negundo L. against cisplatin-induced nephrotoxicity was produced by antioxidant and free radical scavenging activities of flavonoids and alkaloids contents present in the leaf extract of this plant [36].

\section{CONCLUSION}

From this study, this plant was proved as medicinal plant due to the presence of various active principles in their leaf extract. Animal study also revealed that the silver nanoparticles of $V$. negundo $\mathrm{L}$. were profound significant effect in protecting renal damage than $V$. negundo L. along with cisplatin alone, which can be attributed to the slow and regular release of active principles of nanoparticles provided by $V$. negundo L. Thus, this plant could be considered for the preparation of nephroprotective drugs. Further study is required to reveal the protective efficacy of silver nanoparticles synthesized from methanolic leaf extract of $V$. negundo L. in patients with kidney failure who are undergoing chemotherapy with cisplatin.

\section{ACKNOWLEDGMENTS}

The authors would like to thank CECRI, Karaikudi, and Dr. T. Subbao Rao, Special diagnostics, Coimbatore, for providing necessary facilities and support to carry out the research work.

\section{AUTHORS' CONTRIBUTIONS}

Author has contributed to the best of his knowledge for the research work carried out in this article.

\section{CONFLICTS OF INTEREST}

The authors declare that there are no conflicts of interest regarding the publication of this article.

\section{REFERENCES}

1. Ferguson MA, Vaidya VS, Bonventre JV. Biomarkers of nephrotoxic acute kidney injury. Toxicology 2008;245:182-93.

2. Meyer KB, Madias NE. Cisplatin nephrotoxicity. Miner Electrolyte Metab 1994;20:201-13.

3. Appenroth D, Frob S, Kersten L, Splinter EK, Winnefeld K. Protective effects of Vitamin $\mathrm{E}$ and $\mathrm{C}$ on cisplatin nephrotoxicity in developing rats. Arch Toxicol 1997;71:677-83.

4. Clark JS, Faisal A, Baliga R. Cisplatin induces apoptosis through the ERK-p ${ }^{66}$ shc pathway in renal tubule cells. Cancer Lett 2010;297:165-70.

5. Choudhury D, Ahmed Z. Drug-associated renal dysfunction and injury. Nat Clin Pract Nephrol 2006;2:80-91

6. Nordberg G, Gerhardsson LS. Silver. In: Seiler HG, Sigel H, Sigel A, editors. Handbook on Toxicity of Inorganic Compounds. New York: Marcell Dekker Press; 1988. p. 619-24.

7. Hill WR, Pillsbury DM. Argyria: The Pharmacology of Silver. $18^{\text {th }}$ ed. Baltimore: Williams and Wilkins Company Publishers; 1939.

8. Uppalapathi L, Rao JT. Antimicrobial properties of the essential oil of Vitex negundo. Indian J Pharm Sci 1979;50:57-8.

9. Masilungan VA. Anticancer activity of Vitex negundo extracts. Indian J Pharm 1963;25:381

10. Perumalsamy R, Singamuthu S, Sen A. Screening of 34 Indian medicinal plants for antibacterial properties. J Ethnopharmacol 1998;62:173-82.

11. Indhumathi $\mathrm{T}$, Rajathi $\mathrm{K}$. A study on synthesis of silver nanoparticles from Murraya koengii leaf and its antifungal activity. Int J Drug Dev Res 2013;5:293-8.

12. Abd El-Rahman SN, Al-Jameel SS. Protection of curcumin and curcumin nanoparticles against cisplatin induced nephrotoxicity in male rats. Sch Acad J Biosci 2014;2:214-23.

13. Sinha SN, Paul D, Halder N, Sengupta D, Patra SK. Green synthesis of silver nanoparticles using fresh water green alga Pithophora oedogonia (Mont) wittrock and evaluation of their antibacterial activity. Appl Nanosci 2015;5:703-9.

14. Kumari AS, Venkatesham M, Ayodhya D, Veerabhadram G. Green synthesis, characterization and catalytic activity of palladium nanoparticles by xanthan gum. Appl Nanosci 2015;5:315-20.

15. Bobby MD, Wesely EG, Johnson M. FTIR studies on the leaves of Albizia lebbeck benth. Int J Pharm Sci 2012;4:293-6.

16. Maheswari C, Mariyammal R, Venkatnarayanan R. Renal protective activity of Orthosiphon stamineus leaf extract against cisplatin induced renal toxicity. Int J Pharm Technol 2011;1:1584-92.

17. Guguloth S, Vivekanandan L, Singaravel S. Nephroprotective activity of Vitex negundo linn bark against chemical induced toxicity in experimenal rats. Int J Adv Pharm Res 2011;6:462-70.

18. Marsh WH, Fingerhut B, Miller A. Non protein nitrogen, urea, creatine and creatinine. In: Varley $\mathrm{H}$, editor. Practical Clinical Biochemistry. London: William Heinemann Press; 1980. p. 456-68.

19. Ito A. The exercise prescription for hyperuricemia. Nihon Rinsho 2000;58:431-6.

20. Brod J, Siorta JH. The renal clearance of endogenous creatinine in man. J Clin Invest 1948;27:645-54.

21. Beuge JA, Aust SD. The thiobarbituric acid assay. Methods Enzymol 1978;52:306-7.

22. David M, Richard JS. In: Bergmeyer J, Grab M, editors. Methods of Enzymatic Analysis. Deer Field Beach-Florida: Verlag Chemie Press; 1983. p. 358

23. Ochei J, Kolhatkar A. Medical Laboratory Science (Theory and Practice). $2^{\text {nd }}$ ed. New Delhi: Tata McGraw Hill Publishers; 2000.

24. Gupta SP. Textbook of Statistical Methods. $43^{\text {rd }}$ ed. New Delhi: Sultan Chand and Sons Publishers; 2014.

25. Kalainila P, Subha V, Ernest R, Sahadevan R. Synthesis and characterization of silver nanoparticle from Erythrina indica. Asian J Pharm Clin Res 2014;7:39-43.

26. Shankar SS, Rai A, Ahmad A, Sastry M. Rapid synthesis of Au, Ag and bimetallic Au core Ag shell nanoparticles using Neem (Azadirachta indica) leaf broth. J Colloid Interface Sci 2004;275:496-502.

27. Pawar S, Kamble V. Phytochemical screening, elemental and functional group analysis of Vitex negundo L. leaves. Int J Pharm Pharm Sci 2017;9:226-30.

28. Amudha M, Krishnakumari S. Biosynthesis and characterization of silver nanoparticles using the aqueous extract of Vitex negundo L. World J Pharm Pharm Sci 2014;3:1385-93.

29. Shemesh O, Golbetz H, Kriss JP, Myers BD. Limitations of creatinine as a filtration marker in glomerulopathic patients. Kidney Int 1985;28:830-8.

30. Meessen JH, Petersen H. Urea, in Ullmann's Encyclopedia of Industrial Chemistry. $6^{\text {th }}$ ed. Weinheim, Germany: Wiley-VCH Press; 2005.

31. Cirillo P, Sato W, Reungjui S. Uric acid, the metabolic syndrome and renal disease. J Am Soc Nephrol 2006;17:S165-68.

32. Ostrea EM, Cepeda EE, Fleury CA, Balun JE. Red cell membrane lipid peroxidation and hemolysis secondary to phototherapy. Acta Paediatr Scand 1985;74:378-81

33. Deponde M. Glutathione catalysis and the reaction mechanisms of glutathione-dependent enzymes. Biochim Biophys Acta 
2013;1830:3217-66.

34. Mohamed ER, Abdel AH. Antioxidant and anti-inflammatory activities of silver nanoparticles biosynthesized from aqueous leaves extracts of four Terminalia species. Adv Nat Sci Nanosci Nanotechnol 2014;5:1-11.

35. Wahdan SA, Azab SS, Elsherbiny CA, Demerdash EE. Piceatannol ameliorates cisplatin-induced histological and biochemical alterations in rats kidney. Int J Pharm Pharm Sci 2017;9:305-11

36. Gautam LM, Shrestha SL, Wagle P, Tamrakar BM. Chemical constituents from Vitex negundo (Linn.) of nepalese origin. Sci World 2008;6:27-32. 